\title{
Improving the availability of intra-operative images created during trauma operations - a multidisciplinary team collaboration
}

\author{
Thomas Hayes, Edward Harrison \\ Croydon University Hospital
}

\begin{abstract}
Intraoperative $\mathrm{x}$ rays are regularly taken on multiple occasions during orthopaedic surgery, especially during the course of planned trauma lists where procedures are performed under $x$ ray guidance. These images have an important role in demonstrating the nature of the operation to clinical staff and in permitting an assessment of the standard of surgery by colleagues at planned metalwork reviews. Furthermore, they represent an important medico-legal record of the fracture configuration at the time of completion of surgery.

Various technologies are available for the creation, storage, and presentation of such images. In our hospital, relatively unsophisticated technology is employed to physically print such $\mathrm{x}$ rays before they are digitally uploaded to an electronic computer system to be stored. This system also allows the images to be viewed. Unfortunately, many opportunities existed within our systems which created opportunities for images to be lost so that they were not made available to clinical staff. We aimed to evaluate and improve this system with the aim of making all $x$ rays taken intraoperatively available to clinical staff in a timely manner. By examining the processes through which images were handled, we were able to adopt strategies to ensure the prompt production of images.
\end{abstract}

\section{Problem}

$X$ rays are routinely taken at various stages of orthopaedic operations to assist the surgeon and confirm satisfactory reduction and fixation of fractures. In our hospital, such images are taken with an $\mathrm{x}$ ray machine before being physically printed out onto plastic. The subsequent plastic image is then electronically scanned to produce a digital image which is uploaded to our computer system and saved so that it can be displayed and accessed on an electronic database known as the picture archiving and communication system (PACS). While radiographers were physically able to expose patients to radiation, create images, and print them without a written order from a doctor, they were not able to digitise the image unless such an order existed.

We were finding that many of our images were not being made available to us after the operation. The availability of these images is important both clinically and medico-legally. Consequently, it was not possible to confirm adequate fixation, something important to maintain appropriate oversight of the standard of surgery. Such oversight constitutes a particularly important tool for training and development as it permits feedback on the standard of surgery to junior trainees by senior colleagues. In addition, should a complication arise or surgery be unsuccessful then the intraoperative images provide confirmation of the satisfactory reduction and fixation of the fracture at the time the patient left the operating theatre.

\section{Background}

To the author's knowledge, no similar reports exist on BMJ Quality. Searches performed for the terms "x ray" and/or "intraoperative" or "intra-operative" return no appropriate articles. Similarly, no similar quality improvement projects were identified as listed on PubMed, Google Scholar, or Ovid/Medline. Given this, we could identify no evidence as to how best to improve our practice and so adopted our own approach based on the PDSA (plan, do, study, act) model.

\section{Baseline measurement}

Using a plan, do, study, act (PDSA) approach we were able to examine the systems via which intra-operative images from orthopaedic theatres were handled in our hospital. We aimed to identify how many images were taken, and the proportion of these which were not available on the picture archiving and communication system (PACS) within 72 hours of them being taken. A 72 hour deadline was implemented as the orthopaedic department has a once weekly "metalwork" meeting review of all the previous weeks operating every Wednesday. If images are processed within 72 hours it allows for all work done on planned trauma lists (Monday to Saturday, 9:00am to $5: 00 \mathrm{pm}$ ) to be reviewed the following week. It was felt that this was important because on occasions where images were not reviewed at the meeting immediately following from their operation there was increased scope for the patient to be discharged without review of their films (important as satisfaction with fixation may influence postoperative instructions eg weight-bearing status) and a greater risk that images not reviewed at the appropriate meeting may be forgotten about and not reviewed at all.

Having identified if an image was processed fully within 72 hours, we then aimed to identify the cause for occasions where this was not done. Having investigated the cause of delays, two large contributions were identified: 
1. That no written request for the images existed and therefore they could not be processed after being taken. The electronic uploading of images is dependent on an electronic order being made and in the absence of this the images could not be uploaded to the computerised image database.

2. That in the presence of an appropriate electronic order, images were not being digitally scanned and uploaded.

We ran our project's first cycle over a two week cycle. Twentyseven patients were included. In nine (33\%) cases the image was available within 72 hours of the operation. Of the remaining 18 cases, in four (15\%) the cause of delay was identified as a failure to request the image. In 14 (52\%) cases, images correctly requested were not processed satisfactorily.

See supplementary file: ds5508.jpg - "revised graph"

\section{Design}

Examination of the process by which images were created in our trust revealed that the uploading of images onto PACS in a timely manner could be interrupted at three different stages during their production process:

1. Images could not be processed because they had never been requested

2. Images that had been requested were not physically being printed out

3. Physical print outs were not being digitally uploaded.

Our first cycle revealed that in $33 \%$ of cases the delay to the creation of images could be attributed to the first cause. Similarly, after discussion with our radiology department it became apparent that images were routinely physically printed but that difficulties in uploading onto the digital system existed. The chief reasons provided for this was ambiguity as to which images needed to be uploaded due to inconsistent requesting by the operating doctors and a lack of urgency in the minds of the radiographers in processing such images. It became clear over the course of our interactions with the radiographers that the purpose of these images and the fact they were reviewed weekly was not fully understood.

As a consequence of this, two separate changes to policy were adopted to address both causes of delay. In the first instance, we aimed to reduced the number of $x$ rays being performed which could then not be digitally uploaded due to a lack of a valid request to zero. Doctors and radiographers were reminded of their obligations relating to the release of radiation and the need for a valid written order in advance of this. Radiographers were told not to perform intra-operative $\mathrm{x}$ rays in the absence of such a request, even if this delayed operating. We also adopted our trauma list submission template to include a box which medical staff could tick once such an order had been made. This was intended to act as a visual prompt to remind them of the need to do this.

Secondly, we agreed with the radiology department that all images should be uploaded in a timely manner, specifically within 72 hours of the time the image was initially taken. Radiographers were invited to the orthopaedic trauma meeting so that they could appreciate the importance of such images and the risks associated with them not being available for review. In this manner we aimed to increase knowledge across the multidisciplinary team of each professional's contribution to the health and well-being of patients and their contribution to patient care. Through this we hoped that the intervention would be sustainable in the long term due to widespread "buy in" to the proposed changes across the multidisciplinary team.

\section{Strategy}

We undertook two cycles of intervention. Having identified the problem, namely that images were not being made available to clinical staff, we aimed to analyse the processes by which they were processed to identify factors along this process where interruptions were occurring. We then identified where problems were arising and adopted strategies to change behaviour.

It was our intention to target both the occasions where our initial measurements of activity over a two week period suggested impediments to the processing of images occurred. This was undertaken through the adoption of changes in policy communicated to all stakeholders via email. Furthermore, these policy changes were verbally delivered at departmental governance meetings to which all stakeholders both in radiology and surgery were invited. These activities took place over the course of a week. Two weeks were then allowed to pass before the audit was repeated post intervention.

A key part of our intentions was to deliver a sustainable improvement. Consequently, we repeated our audit six weeks after the first audit cycle was undertaken to assess whether or not there had been a sustained change in performance or whether or not any affect was transitory.

\section{Results}

We measured the proportion of images which were not available on PACS within 72 hours from all cases on the planned orthopaedic trauma lists over a two week period. We performed firstly this for our initial baseline measurements, before repeating the assessment two weeks after the implementation of our interventions, and again six weeks post. Where images were not processed adequately, we sought to identify the cause of the delay. Results are demonstrated in the table below.

During our first audit cycle, again performed over two weeks, 15 patients were operated on. The number of cases in which images were not appropriately uploaded and made available fell from $66.0 \%$ to $13.3 \%$. Of the two patients in whom images were not expediently uploaded, one was due to a failure to request the imaging, and a further due to a failure to process images adequately. 


\section{BMJ Quality Improvement Reports}

During the second cycle the improvement seen previously was maintained. Of 20 patients operated on over the second two week period, $90 \%$ were processed within 72 hours. Delays due to a failure on behalf of the orthopaedic team to request images were eliminated entirely.

See supplementary file: ds5509.png - "table revised"

\section{Lessons and limitations}

The most important lesson learnt was the need for broad stakeholder engagement, particularly when the problem being addressed involved separate teams with different priorities and a poor understanding of each others contribution to delivering patient care. It was only once the radiology department were fully engaged and committed that we were able to deliver a sustainable improvement in results.

\section{Conclusion}

We enjoyed significant success in improving the availability of intraoperative images from our planned orthopaedic trauma list cases. The number of images which were not available for review by doctors within 72 hours of being made fell substantially. This permitted their timely review in the immediate post operative phase and should serve to reduce the number of images lost entirely with potentially severe medico-legal consequences. By adopting a multidisciplinary team approach and involving all stakeholders, we were able to introduce sustained and sustainable change which will prove beneficial for patient care.

\section{References}

None.

\section{Declaration of interests}

Nothing to declare.

\section{Acknowledgements}

Mrs Cynthia Walters - superintendent radiologist.

\section{Ethical approval}

Ethical approval was not sought as the study was deemed an improvement study. It was not required by local policy. The study is an audit of local practice regarding the processing of intra-operative $x$ rays. 